\title{
LA TEORÍA DE LA TONALIZACIÓN Y LA DE VALORACIÓN: DOS VISIONES COMPLEMENTARIAS
}

\author{
(Tonalization and appraisal theories: \\ two complementary visions)
}

\author{
María Laura Pardo ${ }^{1}$ \\ (Universidad de Buenos Aires - UBA)
}

\begin{abstract}
The purpose of this study is to analyse two theories: 1.the "tonalization" theory (Lavandera, 1986, Pardo, 1996) and 2. the appraisal theory (Iedema et al, 1994, Martin 2000 and White 2003). Both explore phenomena of the so-called "evaluation", "appreciation" or "tonalization." In order to compare both theories an example is analyzed and differences and similarities are pointed out which also imply different cultural perspectives, as one comes from the West and the other from the East.
\end{abstract}

Keywords: Tonalization theory, Appraisal theory, Theme and Rheme.

\section{RESUMEN}

El propósito de este trabajo es analizar dos teorías: 1. la de la tonalización (Lavandera, 1986, Pardo, 1996) y 2. la de la valoración (Iedema et al, 1994, Martín 2000 y White 2003). Ambas exploran los fenómenos de lo que se denomina "evaluación", "apreciación" o "tonalización". Con el fin de compararlas se analiza un ejemplo y se marcan diferencias y similitudes

1. Directora del Departamento de Lingüística del CIAFIC-CONICET. Investigadora independiente del CONICET. Profesora Adjunta a cargo de cátedra de Análisis de los lenguajes de los medios masivos de comunicación en la Facultad de Letras de la Universidad de Buenos Aires. Directora del UBACYT F127 Análisis crítico del discurso de la pobreza extrema urbana en la Argentina y America Latina en la posmodernidad: una aproximación a la red latinoamericana al análisis crítico del discurso de la pobreza. Miembro fundador de la Red latinoamericana al análisis crítico del discurso de la pobreza (REDLAD). 
de estas aproximaciones que implican también miradas distintas en el plano cultural, ya que una proviene de occidente y la otra de oriente.

Palabras-clave: Teoría de la tonalización, teoría de la valoración, Jerarquización de la información

\section{Introducción}

El objetivo de este trabajo es analizar dos teorías como la de la tonalización (Lavandera, 1986, Pardo, 1996) y la de la valoración (Iedema et al, 1994, Martín 2000 y White 2003) que, en mi opinión, son complementarias y que unidas podrían dar cuenta mejor de los fenómenos relacionados con lo que se denomina "evaluación", "apreciación" o "tonalización”.

La visión que plantea la teoría de la tonalización es mucho más general ya que observa a los recursos que sirven para mitigar o reforzar ciertos aspectos evaluativos en el discurso como parte del principio de la Jerarquización de la Información. Estos recursos mitigadores y reforzadores, por lo tanto, son de carácter obligatorio en el texto y están necesariamente ligados al género discursivo.

Estas miradas sobre el lenguaje y los textos revelan, además, cuestiones de índole cultural, en tanto la teoría de la tonalización es de origen latino y la de la evaluación pertenece al mundo anglo. Como sostiene Shi-xu (2008), esta coocurrencia de formas o maneras de interpretar un fenómeno, sin generalizar sus apreciaciones y complementando vías de análisis lingüístico, enriquece el alcance de una posible teoría que unifique ambas perspectivas.

\section{La tonalización}

Durante años he estudiado el fenómeno de la Jerarquización de la información. Mi investigación estuvo dedicada a extender las 
nociones de tema y rema al texto, que habían sido definidas por la escuela de Praga en relación con la oración. De este modo, dichas nociones, que habían sido estudiadas en proposiciones lógicas y no en emisiones (oraciones puestas en uso), fueron reelaboradas y redefinidas para dar cuenta de las emisiones reales, extensas, tal como se dan en una conversación o en un texto escrito.

Dado que todas las lenguas jerarquizan la información con que los hablantes se expresan, nos encontramos antes un principio del lenguaje. Esto implica que no podemos dejar de "valorar" los argumentos que con que nos expresamos de uno u otro modo. La primera forma de jerarquizar es la que responde al orden de palabras de cada lengua. Dicho orden, ya de por sí, fuerza un tipo de valoración, según la información ocupe el rol de Sujeto o de Predicado.

La jerarquización (y valoración) de la información puede observarse tanto en un plano macro del texto o del discurso en el que pueden reconocerse nociones como las de Tema y Rema del Texto (Pardo, 1992, 1996) o bien Tópico y Comento (van Dijk, 1980) o en un plano micro, es decir al interior de las emisiones como tema, rema y foco de las emisiones. En ambos planos lo que está tematizado se supone menos relevante que aquello que se constituye en la información nueva o remática (lo que el hablante intenta llegar a decir en cada emisión o a lo largo de todo su texto). Además, en ambos se desarrolla la teoría de la tonalización.

La tonalización es un principio del lenguaje, en tanto, señala que todas las lenguas manejan grados de jerarquización iguales o menores al concepto de Tema y Rema, Tema y Rema Textual, Tópico-Comento y es dependiente del género discursivo y del cotexto.

En general los recursos lingüísticos mediante los que se realiza son denominados mitigadores (cuando precisamente mitigan o debilitan un argumento) o reforzadores (cuando lo enfatizan).

La tonalización puede metaforizarse como un sube y baja en el que siempre que algo se mitiga algo se refuerza necesariamente 
y viceversa. De este modo, los conceptos binarios de tema y rema de las emisiones, los textuales, los focos, etc, conllevan de por sí una valoración que puede estar reforzada o mitigada por el uso de otros recursos lingüísticos que aparecen coocurriendo con estos. La tonalización varía según sean sus constituyentes inmediatos, su cotexto y las opciones paradigmáticas (en el sentido de De Saussure) que posea la lengua.

El término tonalización proviene de la pintura como arte y señala cómo puede utilizarse una gradación de colores que va del blanco al negro, con un sinfín de grises en el medio. O sea todo está tonalizado, en otras palabras, todo de alguna forma tiene un grado de valoración. La tonalización puede observarse en un plano micro que apunta a lo gramatical y en lo macro a lo argumentacional.

Por su parte, la teoría de la valoración (Hood y Martin, 2005) se propone describir y explicar los sistemas de opciones semánticas (léxico-gramaticales) que el lenguaje ofrece -y que son utilizados por los hablantes y autores de textos- para evaluar, adoptar posiciones, construir personas textuales o identidades discursivas, asumir roles, negociar relaciones, y transformar en "naturales" las posturas intersubjetivas, que son, en última instancia, ideológicas.

Desde el punto de vista de la teoría de la tonalización, la de la valoración sería una teoría que describe y explica, especialmente el plano léxico-gramatical, cómo el hablante jerarquiza (valora, evalúa) la información que dice o escribe. Mirado desde esta perspectiva, gran parte de esa valoración, en un punto, es obligatoria puesto que nadie puede hablar sin jerarquizar lo que es su decir, por ser este un principio del lenguaje y su realización puede ser conciente o inconciente. Sin embargo, la teoría de la valoración parecería poner énfasis en los aspectos más concientes y creativos del uso, aquellos que no están ni tan sujetos al género discursivo, ni a posiciones configuradas por la lengua.

Veamos un cuadro de síntesis: 


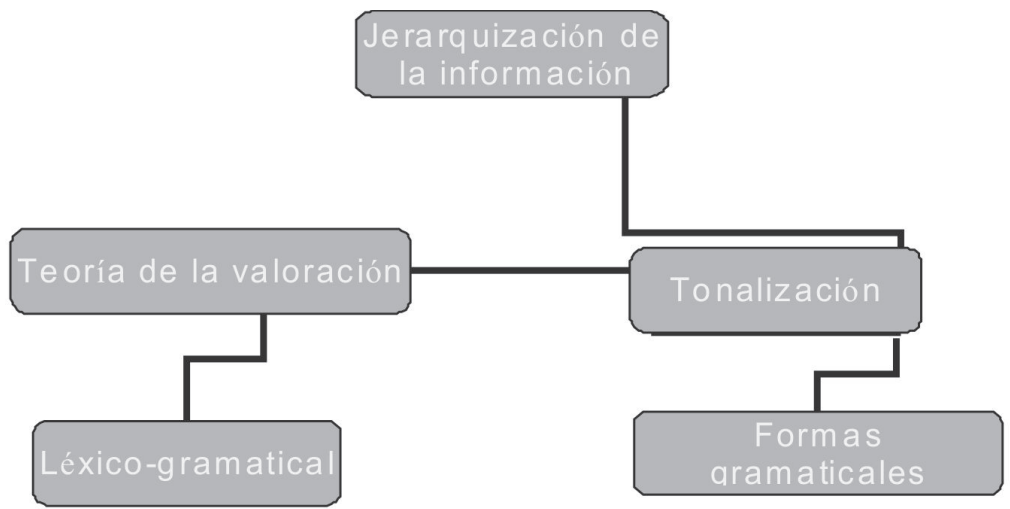

Observamos en el cuadro cómo tanto la teoría de la tonalización como la de la valoración son modos en los que se expresa el principio de Jerarquización de la Información. Parecería que la teoría de la valoración está más ligada a lo léxico gramatical y la teoría de la tonalización a lo gramatical

Lavandera (1986) trabajó especialmente con los recursos mitigadores. En 1996, reveo esta teoría que había quedado inconclusa. Desde entonces he tratado de dar cuenta no solo de los reforzadores, que no habían sido descriptos por ella, sino también de las variaciones que se producen en los diferentes géneros discursivos en relación con este fenómeno.

\section{El plano micro}

Dependiendo del género discursivo, esta jerarquización o tonalización está marcada por el uso contrastivo entre las personas verbales (primeras versus terceras), el Modo (indicativo versus subjuntivo), las voces (activas versus pasivas), etc. Todos estos recursos mitigadores o enfatizadores (en lo que hace a la valoración) son relativos, por lo que los ejemplos que mostraré solo sirven como modelos muy generales, ya que, como decía antes, la valoración varía según el co-texto y el género discursivo en el que aparece. Por ejemplo, en una historia de vida el uso de la primera persona del singular es el uso 
esperable, sin embargo si co-ocurre en el texto con una tercera persona del singular se volverá reforzadora, y paradigmáticamente podría haber tenido el mismo valor reforzador frente al uso de un impersonal. De este modo, se trasciende el plano de lo semántico y lo léxico.

En los textos legales, por ejemplo, la forma estándar es la de la tercera persona del singular por el género (no se utiliza la primera persona en el texto legal), y se tornará reforzadora frente al uso cotextual de los verboides y frente a la posibilidad paradigmática (en el sentido de De Saussure) de un impersonal mantendrá su valor reforzador. En el caso excepcional de un uso de primera persona en el texto legal, desafiando al género, se tornará mitigadora frente a dicho uso. Por lo que son pocos los recursos lingüísticos (no léxicos) que son per se reforzadores o mitigadores.

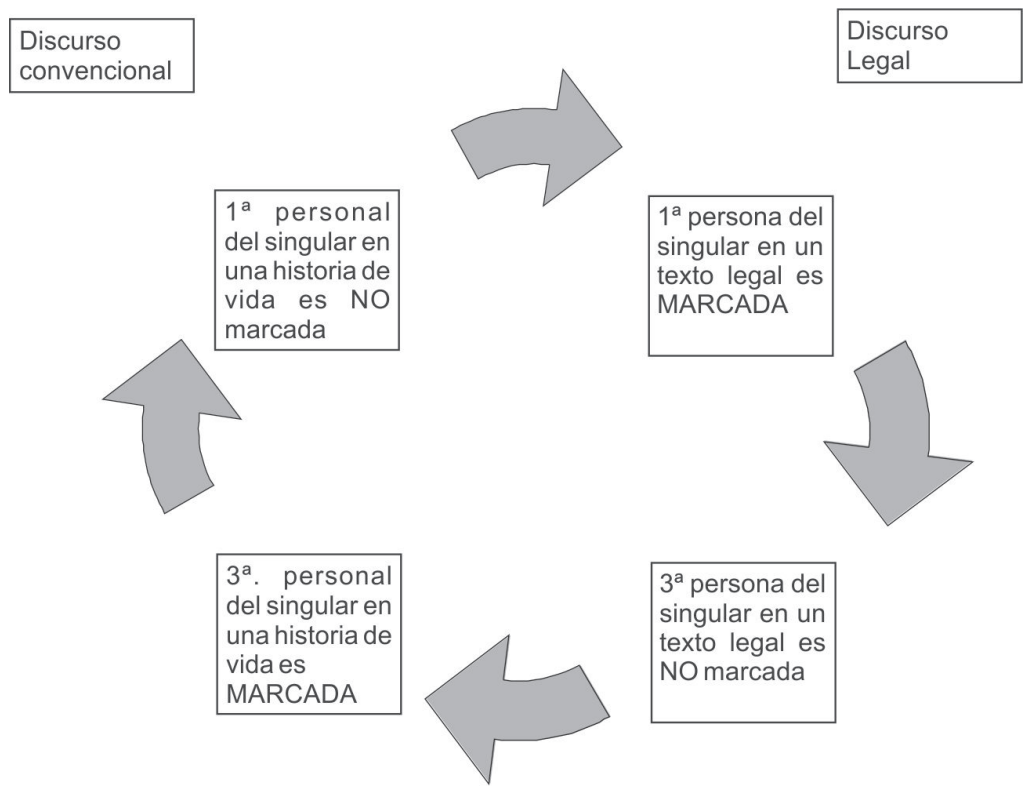

De modo muy genérico - y sin que esto signifique que estas nociones y ejemplificaciones son invariables - podemos decir que algunos de los recursos mitigadores y reforzadores pueden observarse de la siguiente forma en un género conversacional. 


\section{Discurso conversacional}

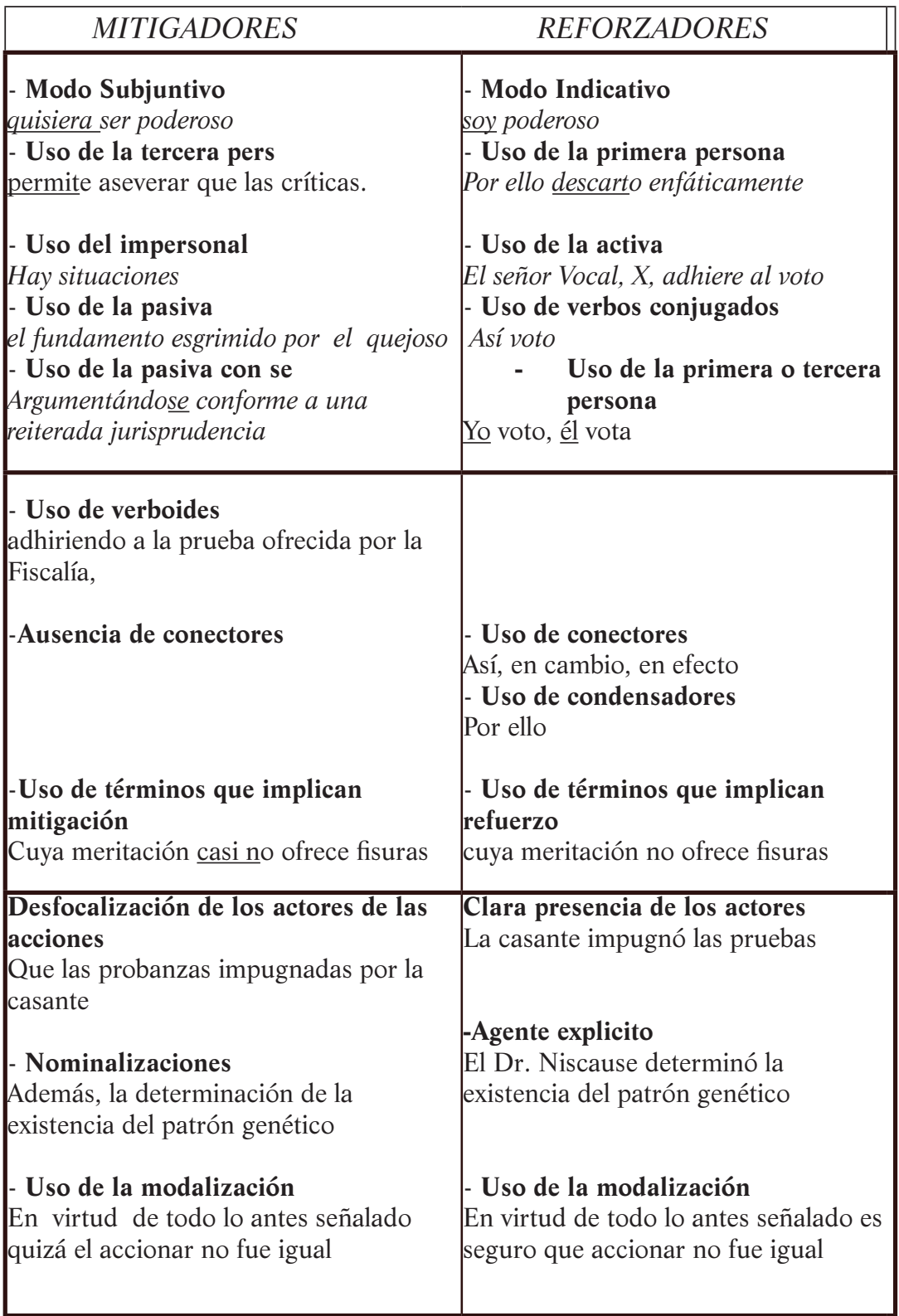


Ahora bien si cambiamos de género y vamos al discurso legal, podemos ver cómo algo que antes era mitigador, como el Modo subjuntivo, se hace reforzador frente al uso de verboides, como la tercera persona del singular que antes (en el género conversacional) era mitigadora es reforzadora frente al impersonal, como el uso de la pasiva es ahora reforzador frente al uso de verboides y construcciones de meta con valor de futuro y cómo la deslocalización de los actores es más reforzadora que la nominalización.

\section{Discurso Legal}

\begin{tabular}{|l|l|}
\hline - Uso de verboides & - Modo Subjuntivo \\
- Uso del impersonal & - Uso de la tercera pers \\
$\begin{array}{l}\text { - Uso de verboides y de construcciones } \\
\text { de meta con valor de futuro (para } \\
\text { construir) }\end{array}$ & - Uso de la pasiva \\
- nominalizaciones & $\begin{array}{l}\text { - Desfocalización de los actores de las } \\
\text { acciones }\end{array}$ \\
\hline
\end{tabular}

Por lo que todo lo que se valora, sea mitigando o reforzado varía según su cotexto y el género discursivo en el que se encuentra inserto.

Tanto para la teoría de la valoración como para la de la tonalización la dimensión dialógica es fundamental. Mientras que la primera hace hincapié en la intertextualidad y el intercambio, la segunda se apoya en la argumentación y en el aporte de justificaciones mediante datos: externos o "internos", entendiendo por estos últimos aquellos que tienen que ver con las emociones del hablante (afecto, juicios). En este sentido, la teoría de la valoración aparece como un aporte que permite una mejor descripción de aquellos datos internos de la teoría de la tonalización.

Veamos un ejemplo comparativo de análisis que aparece en White (2003:3), traducido por mí, que circula masivamente en nuestro país: 
There is an argument, though, is there [atribución], the banks have been a bit [gradación: fuerza] greedy [actitud] I mean, the prdeits are high and good on them [actitud], they're entitled to have high prdeits, but at the same time the fees are bodering on [gradación: foco] the unreasonable [actitud] now.

Sin embargo, allí hay un argumento [atribución], los bancos han sido un poco [gradación: fuerza] ambiciosos [actitud: juicio] quiero decir, las ganancias son altas y aceptables para ellos [actitud: juicio], ellos están facultados a obtener mejores beneficios, pero al mismo tiempo los impuestos ahora están lindando con [gradación: foco] lo disparatado [actitud: juicio]

Un análisis de la tonalización daría este primer resultado:

Sin embargo es un conector que acciona como 'el pero' poniendo un límite a la emisión anterior y [reforzando] lo que sigue de la emisión que encabeza.

Alli: un adverbio de lugar indeterminado que podría mitigar dado que indica alejamiento, pero que en este caso [refuerza] al verbo impersonal.

Hay: forma impersonal que [mitiga] a los actores que sostienen dicho argumento.

Una opción no mitigadora sería:

Sin embargo, muchas personas tendrían un argumento acá...

En este caso, la co-ocurrencia señala que el hablante busca, por un lado, reforzar su idea de que hay un argumento "contra los Bancos”, pero que a la vez no quiere tomar una posición personal frente a esto (de ahí las mitigaciones)

Los Bancos: es un mitigador frente al uso paradigmático de actores personales concretos. 
Han sido: es un verbo en pasado que se contrapone con el presente, tornándose por esta oposición en mitigador

un poco: es un lexema mitigador frente a la fuerte carga semántica que tiene el lexema al que acompaña

ambiciosos: (aquí es donde la tonalización solo diría que este es un lexema que tiene una carga semántica reforzadora de la acción del Banco), en la Teoría de la Valoración (TV de aquí en más), en cambio, sería una [actitud]

quiero decir: quiero es un modal [reforzador] del decir en tanto es una primera persona que se contrapone al uso de la tercera de la cláusula anterior

las ganancias: en este caso serían lexemas positivos

altos: lexema [reforzador], en mi opinión sería en términos de la TV [gradación: fuerza]

aceptables: lexema reforzador, en la teoría de la valoración sería [actitud]

Tanto los bancos y el uso del para ellos: mitiga y esconde a quienes son los banqueros, los actores que toman las decisiones de los "BANCOS". Este uso del sustantivo se opone paradigmáticamente al de una persona gramatical

ellos: pronombre personal de tercera del plural pero cuyo referente son los bancos

Hay que notar que el uso de la repetición que es reforzadora.

están facultados a obtener: uso del presente de indicativo combinado con una construcción de meta que indica en el español rioplatense una acción futura 
mejores beneficios: reforzador de gradación

pero: reforzador limita y anticipa la emisión

al mismo tiempo: circunstancial de tiempo - reforzador del argumento del hablante

ahora: circunstancial de tiempo - reforzador del argumento del hablante

lindando con [gradación: foco]: reforzador de la igualdad temporal

lo disparatado [actitud]: lexema carga semántica reforzadora del argumento del hablante

Ahora bien qué se mitiga y qué se refuerza. Se mitigan y refuerzan argumentos, en pos del sostén de una tesis. De la misma forma, se utilizan actitudes (afectos, juicios, apreciaciones), gradación (fuerza y foco) y compromiso para hacerlo.

\section{Análisis de los paradigmas o cadenas argumentativos}

De allí que el ejemplo pueda analizarse como dos paradigmas argumentativos (todos los textos tienen al menos dos paradigmas argumentativos (Lavandera y Pardo, 1987 y Pardo, 2008), el que quiere sostener el hablante y el que refleja las distintas voces con las que dialoga dicho Hablante, que supuestamente intentan rebatir su argumento. De este modo, el hablante se anticipa a posibles vocesargumentos sociales lo que le permite sostener mejor su argumento. ¿Podría dar esto cuenta de lo que se denomina Compromiso dentro del dialogismo y de la heteroglosia que postula la Teoría de la Valoración solo que en un plano más macro?

El argumento del hablante sostiene que más allá de lo que otras voces dicen (y con las que no contamos en este texto) existe un 
argumento. El hablante refuerza el "sin embargo" para dar cabida a su futura explicación, pero mitiga la prueba de que existe el argumento, probablemente, podríamos interpretar que el hablante no quiera ir en contra abiertamente de los bancos o no sepa a ciencia cierta si podrá sostener la existencia de dicho argumento. Aunque me inclino por la primera suposición.

Comienza entonces el Hablante a argumentar sobre los bancos no sobre los banqueros (lo que es una forma de mitigación frente a la posibilidad de nombrar actores) sostiene fuertemente que son ambiciosos, pero mitiga esa afirmación (la gradúa) y se tornan "poco" ambiciosos. A su vez, aclara reforzando, por si el juicio - refuerzo fue mucho (ambiciosos), quiero decir: las ganancias son altas, si bien altas refuerza a las ganancias, las ganancias altas son mitigadoras con respecto a lo ambiciosos que eran, por lo que en el texto accionan mitigadoramente. Esto implica ya un cambio acerca del análisis, sea que se use la teoría de la valoración o la de la tonalización, de cada uno a uno de los recursos en un texto, estos deben analizarse en coocurrencia con su cotexto. Los lexemas aceptables para ellos, son juicios y a la vez refuerzan su argumento anterior: son ganancias altas, no ambiciosas y para ellos esto es aceptable, continúa sí mitigando a los actores ya que ese ellos refiere a los bancos y no a personas reales. Repite el ellos creando un refuerzo del argumento, logrando así una suerte de separación entre ellos y los que no pensamos como ellos, pero a la vez refuerza su argumento en defensa de los bancos diciendo que ellos están facultados para obtener mejores beneficios (dos palabras estas últimas que refuerzan la cadena: ganancias, altas, aceptables y ahora mejores beneficios) para alcanzar un pero que limita (mitiga) todas estas aseveraciones sobre los bancos con un fuerte argumento, que se ancla en una simultaneidad presente: $A l$ mismo tiempo, ahora, lindando. Estas tres formas de referencia al presente contrastan en el texto con el uso del pasado: los bancos han sido, como si estos ya hubiesen dejado de ser aquellos ambiciosos.

Las formas en presente y su reiteración (Ahora, están), buscan, entonces, reforzarse entre sí y al argumento, que finaliza con un fuerte juicio: disparatado, el ítem lexical más fuerte del párrafo, 
juicio que además está en foco (en términos de la jerarquización de la información).

\begin{tabular}{|ll|ll|}
\hline Argumento del $\boldsymbol{H - P}$ & Voces & \\
\hline$\underline{\text { Sin embargo }}$ & reforzador & Los bancos & mitigador \\
Alli & mitigador & Han sido & mitigador \\
Hay & mitigador & Un poco & mitigador \\
& & Ambiciosos & reforzador \\
& & Quiero decir & reforzador \\
& & Son altanancias & reforzador \\
Pero & Aceptables & reforzador \\
Al mismo tiempo & reforzador & Para ellos & mitigador \\
Ahora & reforzador & Ellos & mitigador \\
Están & reforzador facultados & reforzador \\
Lindando & reforzador & Para obtener & reforzador \\
Con lo disparatado & reforzador & & \\
\hline
\end{tabular}

Como puede apreciarse, y tal como lo había mencionado, los recursos mitigadores o reforzadores toman tal carácter según la relación que tengan con los otros elementos con igual función en el texto. Algo que asiladamente puede ser un mitigador, puede ser reforzador respecto de un mitigador de menor grado. Así, podemos decir que los recursos mitigadores o reforzadores no son per se una cosa o la otra, sino que varían según el texto y el género en el que se encuentren. Y esto es fácilmente observable en los paradigmas argumentativos que maneja cada hablante en cada uno de sus textos (orales o escritos). 
Esta visión holística y completa de los paradigmas argumentativos y del funcionamiento de la tonalización son imprescindibles para dar cuenta de la función de dichos recursos en el texto.

\section{Consideracines finales}

Aunque muy brevemente, creemos haber dado un paso en el camino de la comparación de dos teorías que parecen complementarse: la teoría de la tonalización y la de la valoración. La primera, reitero, aparece como más cercana a lo gramatical y la segunda a aspectos léxico-gramaticales. Además, cada una de ellas revela un modo de acercamiento al texto diferente, especialmente, en lo que hace al dialogismo, aunque no son contradictorias, sino que muestran formas, miradas distintas sobre un mismo fenómeno. Es importante, para cualquier aproximación al texto, tener en cuenta los constituyentes inmediatos y mediatos en relación con los recursos lingüísticos mediante los cuales se expresa la valoración, de la misma forma que al género en que se encuentran insertos.

El hecho de provenir de diferentes ambientes culturales enriquece aún más las aportaciones de ambas aproximaciones.

Recebido em março de 2010 Aprovado em abril de 2010 pardo.linguistica@gmail.com

\section{Referencias bibliográficas}

Hood, S. and J. Martin, 2005. Invoking attitude: the play of graduation in appraising discourse, Revista Signos, vol. 38, no. 58, pp. 195-220.

Iedema, R., S. Feez \& P.R.R. White. 1994. Media Literacy, Sydney, Disadvantaged Schools Program, NSW Department of School Education. 
Lavandera, B. 1986. "Decir y Aludir: una propuesta metodológica”. Cuadernos del Instituto de Lingüística 1 (1): 1-14.

Lavandera, B. y M.L.Pardo, 1987. La Negación en el discurso: patrones y rupturas, en: Análisis sociolingüístico del discurso político. Cuadernos del Instituto de Lingüística. Facultad de Filosofía y Letras de la Universidad de Buenos Aires y CONICET.

Martín, J. 2000. Beyond Exchange: Appraisal Systems in English, en: S. Huston y G. Thompson (eds.) 2000. Evaluation in text: Authorial stance and the construction of discourse. Oxford: Oxford University Press, pp. 142-175 Pardo, M.L.1992 Derecho y Lingüística. ¿Cómo se juzga con palabras? I edición. Buenos Aires: Centro Editor de América Latina.

Pardo, M.L.1996. Derecho y Lingüística. ¿Cómo se juzga con palabras? II edición aumentada y corregida. Buenos Aires: Nueva visión.

Pardo, M.L. 2008. Pardo, M. L. 2008. Una metodología para la investigación lingüística del discurso, en: Pardo, M. L. (ed.), 2008. El discurso sobre la pobreza en América Latina. Santiago de Chile: Frasis, Santiago de Chile: Editorial Frasis. ISBN 978-956-8170-14-1, pp.55-79

Shi-xu 2009. Reconstruyendo los paradigmas orientales de los estudios del discurso, en: Revista Latinoamericana de estudios del discurso 7(1): 79-94 van Dijk, Teun A. 1980. Texto y contexto. Madrid: Cátedra.

White, P.R.R. 2003. Beyond modality and hedging: A dialogic view of the language of intersubjective stance, en: Text 23 (2), pp 259-284 